\title{
Medical big data for smart healthcare
}

\author{
Hyung-Jin YOON ${ }^{* 1,2}$ \\ 'Department of Biomedical Engineering, Seoul National University Hospital, Seoul, Korea \\ ${ }^{2}$ Medical Big Data Research Center, Seoul National University College of Medicine, Seoul, Korea
}

Lecture: As most people expect, the COVID-19 pandemic will change our life radically. One of the directions is expected to minimize off-line contacts in daily lives and healthcare is surely not the exception. Big hospitals, which are centralized systems evolved to deliver healthcare most effectively, can become the media of the spread of diseases in some cases and the adoption of a decentralized healthcare system will be a helpful strategy to prevent it. This strategy can be implemented through a smart healthcare system, and it will be one more necessity of a smart healthcare system in our society.

Smart healthcare, which will be delivered mainly through online contact, is a very comprehensive concept enabling otherwise not possible healthcare such as health monitoring using life-log data from the wearable device and far beyond the online consultation with a doctor. Although there will be many social, ethical, and technical challenges for smart healthcare, the discussion today will be limited to two challenges, interoperability and protecting the data subjects' rights. Smart healthcare needs to collect and process comprehensive health information from a vast number of data subjects during its development and delivery and the data subjects' rights are highly vulnerable during these processes. Collecting and exchanging health information from various sources such as hospitals, clinics, smartwatches, and so on, inevitably raises the issue of interoperability of the data from different sources. The implementation of smart healthcare requires technical solutions to both challenges.

General Data Protection Regulation, recently issued by the EU, deals the data subjects' rights very seriously, such as the right to be informed, the right of access, the right to rectification, the right to erasure, the right to restrict processing, the right to data portability, the right to object, and the rights in relation to automated decision making and profiling. Technical innovations required to protect these data subjects' rights will be discussed here.

Compared to traditional healthcare which mostly dealt with medical data generated within the healthcare facility itself, smart healthcare requires health data beyond the healthcare facilities. Not to mention the life-log data from different wearable devices, even the medical data from different hospitals are not interoperable. Although many health information standards have been proposed, such as Systematized Nomenclature of Medicine Clinical Terms (SMOMED CT), Logical Observation Identifier Name Code (LOINC), Health Level 7 Fast Healthcare Interoperability Resources (HL7 FHIR), and so on, the adoption of these standards in hospital information system in Korea has been very slow. This low adoption of health information standards in our hospital information system obstructs the so-called, meaningful use of electronic medical records. The necessity of adopting health information standards will be refreshed today.

Smart healthcare requires the active participation of health consumers and active participation of health consumers requires their health literacy. Health literacy of health consumers will be fostered by innovative technical aids such as data visualization, the decision support system for consumers, and more. Health literacy will the most powerful driving force of smart healthcare implementation. 